\title{
Mother-and-child catheter-facilitated optical coherence tomography: A novel approach to improve intracoronary imaging
}

\author{
Marcos García-Guimaraes, Javier Cuesta, Fernando Rivero, Teresa Alvarado, \\ Gonzalo Navarrete, Teresa Bastante, Amparo Benedicto, Fernando Alfonso \\ Cardiology Department, Hospital Universitario de La Princesa, Madrid, Spain
}

\begin{abstract}
Background: Our aim was to prospectively analyze the usefulness of mother-and-child catheter approach with the GuideLiner ${ }^{\circledR}(G L)$ catheter extension system to overcome some potential limitations associated with the use of optical coherence tomography (OCT).

Methods: We included consecutive patients referred to our institution for coronary angiogram in whom OCT image acquisition was obtained using GL. From February 2015 to May 2016,
\end{abstract} a total of 19 procedures (18 patients) were prospectively included.

Results: In more than two thirds of the procedures, the OCT catheter was unable to cross the coronary stenosis prior to the use of GL. The right coronary artery was the most frequently involved coronary vessel (63\%). The main reason for using GL (47\% of cases) was poor engagement of the coronary ostium and lack of support of the guiding catheter (47\%). Eventually, in 18 out of 19 procedures, a good quality OCT image was obtained and, when indicated, successful percutaneous coronary intervention with stent implantation was performed. Of note, there were no serious complications related to the use of GL during OCT imaging.

Conclusions: Optical coherence tomography through a catheter extension system is a useful technique able to overcome some of the limitations associated with this imaging technique ensuring adequate blood clearance and thus optimal image quality. (Cardiol J 2016; 23, 6: 647-651)

Key words: optical coherence tomography, image quality, percutaneous coronary intervention, complex percutaneous coronary intervention

\section{Introduction}

Optical coherence tomography (OCT) is currently increasingly used in the evaluation of coronary stenoses and during stent optimization. However, clinical experience demonstrates that OCT image acquisition has several limitations, most of which related to the difficulty to advance OCT catheter across excessive angulated vessels, severely calcified lesions, or critical coronary stenoses. Crossing a recently implanted stent may be difficult also in some cases. Moreover, OCT is associated with an increase in contrast volume administration of special concern in patients with impaired renal function at high risk for contrastinduced nephropathy (CIN). Some of these limitations, however, may be overcome with the help of extension catheters, such as the GuideLiner ${ }^{\circledast}$ (GL) (Vascular Solutions, Inc. Minneapolis, USA). GL is a "child" catheter-extension system initially developed to improve stent delivery. Since its Conformité Européene Marking approval in 2009, several additional applications of this device have been reported. For example, GL has proved to

Address for correspondence: Fernando Alfonso, MD, PhD. Departamento de Cardiología, Hospital Universitario de La Princesa, IIS-IP, Universidad Autónoma de Madrid, c/ Diego de León 62, Madrid 28006, Madrid, Spain, e-mail: falf@hotmail.com 
represent a valuable adjunctive tool in the treatment of chronic total occlusions (CTO) and bypass graft-related lesions [1]. Moreover, GL may be of great help in the delivery of rotational atherectomy burr in patients with tortuous vessels [2,3] or even to facilitate coronary thrombus aspiration in the setting of ST-segment elevation myocardial infarction (STEMI) [4, 5].

However, the potential value of GL to facilitate OCT imaging remains to be established. A single case report suggested a possibility of using GL to improve OCT image acquisition [6]. The aim of this prospective study was to systematically evaluate the value of GL during OCT imaging.

\section{Methods}

From February 2015 to May 2016, consecutive patients referred for coronary angiography to our institution in whom OCT image acquisition was possible with a "mother-and-child" catheter approach were included. Clinical and demographic data were prospectively collected. Data related to the procedure were directly extracted from our internal dedicated interventional database. OCT was performed with the Dragonfly OPTIS imaging catheter (St. Jude Medical Inc. MN, USA). The GL V3 5-in-6 catheter was used as a catheter extension system. In all these studies, iodixanol was used as a contrast medium in an automatic injection and standard parameters for OCT acquisition (flow $5 \mathrm{~mL} / \mathrm{s}$; total volume $15 \mathrm{~mL}$ ). CIN was defined as either a $25 \%$ increase in serum creatinine from baseline levels or $0.5 \mathrm{mg} / \mathrm{dL}$ increase in its absolute value, within $48-72 \mathrm{~h}$ after the procedure. In patients in whom GL was used to improve OCT image quality, this parameter was evaluated by a modified scoring system derived from a previous classification described by Capodanno et al. [7]. A zone of interest at the level of the target lesion (10 mm length) was analyzed for both OCT runs recorded with and without the use of GL. These runs were assessed frame by frame according to the following values: 0 - inability to identify vessel wall for the entire circumference; 1 - ability to identify vessel wall at the region of interest only in $90^{\circ} ; 2-$ ability to identify vessel wall at the region of interest only in $180^{\circ} ; 3$ - ability to identify vessel wall at the region of interest only in $270^{\circ} ; 4-$ ability to identify vessel wall at the region of interest in $360^{\circ}$.

The study was approved by the local bioethical committee and all patients gave their informed consent.
Table 1. Clinical and demographic data.

\begin{tabular}{lc}
\hline Age [years] & $66.4 \pm 8.6$ \\
Sex (male) & $17(94 \%)$ \\
Hypertension & $16(89 \%)$ \\
Hypercholesterolemia & $15(83 \%)$ \\
Diabetes & $10(56 \%)$ \\
Smoking/ex-smoker & $11(61 \%)$ \\
Previous MI & $4(22 \%)$ \\
Previous PCI & $10(56 \%)$ \\
Previous CABG & $2(11 \%)$ \\
Indication for study: & \\
Stable angina & $3(16 \%)$ \\
NSTEMI & $9(47 \%)$ \\
STEMI & $2(11 \%)$ \\
Ventricular arrhythmia & $1(5 \%)$ \\
$\quad$ Asymptomatic (protocol) & $4(21 \%)$ \\
Chronic kidney disease & $4(22 \%)$ \\
(GFR < 60 mL/min/1.73 $\left.\mathrm{m}^{2}\right)$ & \\
\hline
\end{tabular}

$\mathrm{MI}$ - myocardial infarction; $\mathrm{PCl}$ - percutaneous coronary intervention; CABG - coronary artery bypass graft surgery; NSTEMI - non-ST-segment elevation myocardial infarction; STEMI ST-segment elevation myocardial infarction; GFR - glomerular filtration rate

Table 2. Procedural data.

\begin{tabular}{lc}
\hline Main indication for GL: & \\
Guiding catheter poor support & $9(47 \%)$ \\
Severe tortuosity & $1(5 \%)$ \\
Severe calcification & $3(16 \%)$ \\
Increase OCT image quality & $6(32 \%)$ \\
Approach: & \\
Radial & $15(79 \%)$ \\
Femoral & $4(21 \%)$ \\
Target vessel: & \\
LAD & $2(11 \%)$ \\
LCX & $3(16 \%)$ \\
RCA & $12(63 \%)$ \\
Saphenous vein graft & $2(11 \%)$ \\
Lesion type: & \\
B1 & $4(25 \%)$ \\
B2 & $3(19 \%)$ \\
C & $9(56 \%)$ \\
Lesion length [mm] & $19.2 \pm 11.7$ \\
Chronic total occlusion & $2(13 \%)$ \\
Stent implantation & $16(84 \%)$ \\
Contrast volume [mL] & $271.5 \pm 108$ \\
Flouroscopy time [min] & $28.2 \pm 19.5$ \\
\hline
\end{tabular}

GL - GuideLiner; OCT - optical coherence tomography; LAD left anterior descending coronary artery; LCX — left circumflex coronary artery; RCA — right coronary artery 


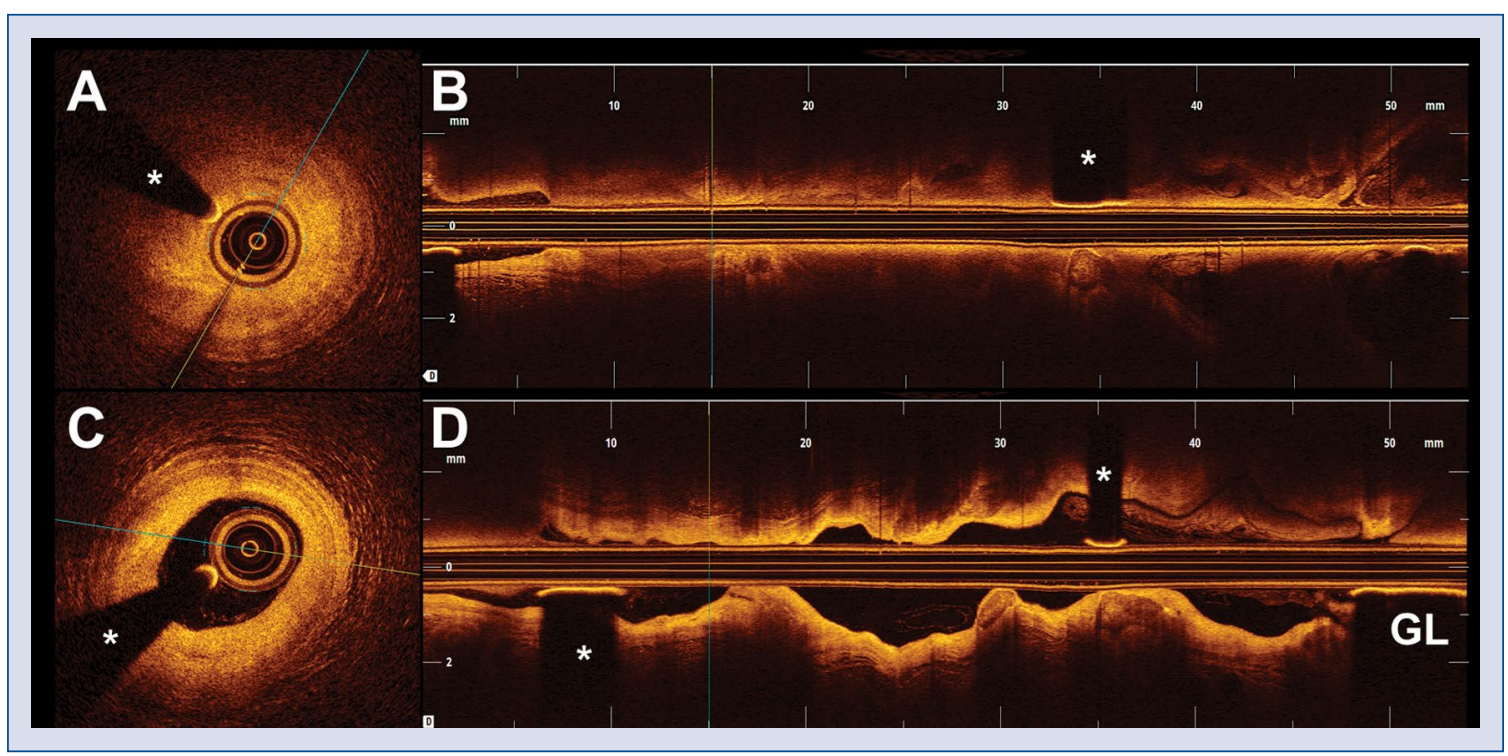

Figure 1. A 79-year-old man was referred for coronary angiogram due to new onset of stable angina. Angiography depicted a moderate lesion at the distal segment of the left circumflex coronary artery (LCX); A, B. Conventional optical coherence tomography (OCT) run throughout the guiding catheter; A. Region of interest with suboptimal quality due to blood artefact; C, D. OCT run throughout the GuideLiner ${ }^{\circledast}(\mathrm{GL})$ extension catheter depicting a predominantly fibrotic lesion at the distal portion of the LCX; C. The lesion was caused by a fibrotic plaque that caused a reduced lumen area; asterisk $\left({ }^{*}\right)$ denotes wire artefact.

\section{Statistical analysis}

Mean value was calculated for both groups and compared using Student's t-test for paired data. The expected number of patients undergoing this diagnostic strategy was too small to permit a formal power calculation. Continuous variables were presented as mean \pm standard deviation and categorical variables as counts and percentages. Statistical analyses were performed with STATA 12 (Statacorp LP).

\section{Results}

During the study period, the GL-OCT strategy was used in 18 consecutive patients (19 procedures). The possibility of using this diagnostic strategy was discussed among our group of interventional cardiologists at the beginning of the study but its use in a given patient was left to the criteria of the operator. Clinical characteristics of the patients are summarized in Table 1. Most patients were male (94\%), with a mean age of $66 \pm 9$ years old. A high prevalence of classic coronary risk factors was found. More than a half of the patients had a history of percutaneous coronary intervention (PCI). The main indication to perform coronary angiography was diagnosis of non-STEMI (47\% of patients). Procedure-related characteristics are summarized in Table 2 . The majority of these pro- cedures $(79 \%)$ were performed with a trans-radial approach (79\%). The most frequent target vessel was the right coronary artery (RCA) (63\%). With the exception of 3 per-protocol scheduled followup diagnostic coronary angiograms, 16 procedures required PCI with stent implantation. Majority of target lesions (56\%) were complex American Heart Association/American College of Cardiology (AHA/ACC) type $\mathrm{C}$ lesions and there were $2 \mathrm{CTOs.}$ In $68 \%$ of the procedures, the OCT catheter was unable to cross the lesion without the use of GL. The main reason for using GL was poor support of the guiding catheter at the coronary ostium or poor engagement of guiding catheter. Only in 1 patient the advancement of the OCT catheter was unsuccessful despite the use of GL. In this patient, adequate support of the guiding catheter could not be obtained on saphenous vein graft that had a severely angulated take-off and a critical proximal lesion. Nevertheless, in these patients, a stent could be eventually implanted with success at the target lesion. Alternatively, in 6 of these procedures, GL was used in an attempt to improve OCT image quality. Of note, adequate OCT image quality was eventually obtained in all patients in whom GL was selected to improve image quality (Figs. 1, 2). In those 6 patients in whom direct comparison of the OCT runs - with and without GL - was possible, (total number of analyzed fra- 


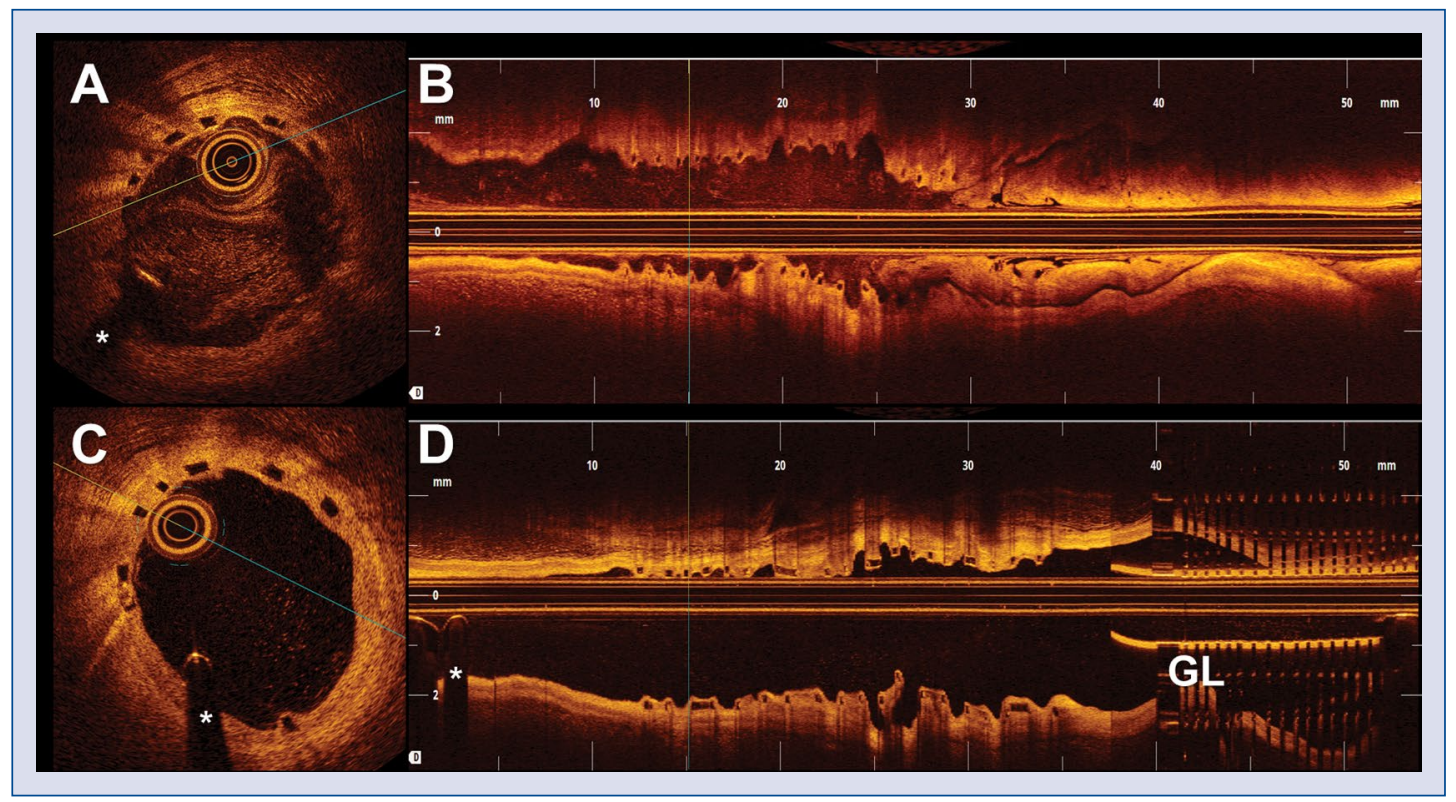

Figure 2. A 70-year-old woman presented an inferior ST-segment elevation myocardial infarction. Coronary angiogram revealed an acute occlusion at the proximal segment of the right coronary artery. A 3.0/18 mm bioresorbable vascular scaffold (BVS) was successfully implanted. A repeated coronary angiography was scheduled - per-protocol -9 months after the index event; A, B. Conventional optical coherence tomography (OCT) run with poor image quality as a result of blood artefact due to poor support of the guiding catheter; C, D. OCT run throughout GuideLiner ${ }^{\circledR}$ $(\mathrm{GL})$ catheter revealing satisfactory mid-term results. The BVS showed adequate expansion, absence of malapposition and a sufficient neointimal coverage; asterisk $\left({ }^{*}\right)$ denotes wire artefact.

mes $=1200)$ image quality assessment by our score improved from a mean of $1.6 \pm 0.4$ without GL to a mean of $3.8 \pm 0.1$ with the use of GL $(p<0.01)$. In addition, in patients with PCI indication, stent implantation was always successful. Notably, there were no serious clinical complications that could be directly attributed to the use of GL in this context. A patient showing a thrombotic occlusion of a severely ectatic RCA experienced proximal thrombus dislodgement after distal contrast injection throughout GL but without any clinical repercussion. Another patient suffered no-reflow phenomenon during the treatment of a lesion in a different vessel from that requiring OCT with the use of GL. No patient developed CIN after the procedure. The relatively large amount of contrast media required during these procedures (Table 2) was in part a result of the attempts to obtain a good vessel visualization before the use of GL.

\section{Discussion}

Optical coherence tomography imaging is helping interventional cardiologists to better understand atherosclerotic coronary artery disease. OCT allows a superb, high-resolution stent visualization and therefore can be used to optimize stent implantation [8]. However, in certain anatomic scenarios (vessels with excessive tortuosity, extremely angulated, severely calcified or critical lesions), crossing the lesion with the OCT catheter may be challenging. In other patients, image quality remains suboptimal as a result of insufficient blood clearance from the target segment. GL is a "child" catheter, initially designed to improve stent delivery by augmenting support and correcting coronary angulations. In this anatomic setting, GL may also help advance the OCT catheter across the target lesion. Importantly, the GL catheter allows a supraselective contrast injection, therefore improving OCT image quality and potentially reducing the total amount of contrast required for optimal imaging. The leakage of contrast at the aortocoronary junction, typically occurring during conventional coronary injections, is completely prevented by the use of GL. This may be of particular benefit in patients with inadequate coronary engagement or in those with poor guiding catheter support.

To the best of our knowledge, this is the first prospective and systematic study addressing the value of GL during OCT imaging. Our findings demonstrate that the use of GL improves image 
quality in most patients with suboptimal images on conventional OCT imaging. Furthermore, this series confirms that this diagnostic strategy can be readily implemented in selected challenging cases. Finally, this diagnostic strategy is simple and safe.

Previous series about the use of GL in other clinical settings have described complications related to the use of this special catheter. Initially, some of them were a cause of concern. These included catheter-induced coronary dissections, stent damage across GL, or episodes of hypotension, or even ventricular arrhythmias provoked by very deep intubation with sub-occlusion of the coronary vessel $[9,10]$. Our data illustrate that with the evolution of GL technology (to the current V3 and XL GL), associated with the acquired clinical experience, most of these complications may be fully prevented. However, the possibility of proximal thrombus displacement leading to systemic embolization should be considered before injecting through a GL deeply advanced into lesions with a large thrombus burden.

\section{Conclusions}

Mother-and-child catheter-facilitated OCT with GL seems to be a useful and safe technique both to help in the characterization and treatment of coronary lesions. This approach was able to provide good-quality OCT images and successful PCI procedures even in patients with challenging anatomic scenarios. Further studies are still required to demonstrate that the systematic use of GL in selected patients may be associated with a significant reduction in the total amount of contrast required for coronary imaging.

Conflict of interest: None declared

\section{References}

1. Kovacic JC, Sharma AB, Roy S et al. GuideLiner mother-andchild guide catheter extension: A simple adjunctive tool in PCI for balloon uncrossable chronic total occlusions. J Interven Cardiol, 2013; 26: 343-350.

2. Costanzo P, Aznaouridis K, Hoye A, Alahmar A. GuideLinerfacilitated rotational atherectomy in calcified right coronary artery: the "child" makes the difference. JACC Cardiovasc Interv, 2016; 9: e47-e48.

3. Vo M, Minhas K, Kass M, Ravandi A. Novel use of the GuideLiner Catheter to deliver rotational atherectomy burrs in tortuous vessels. Case Rep Cardiol, 2014; 2014: 594396.

4. Numasawa Y, Motoda H, Yamazaki H, Kuno T, Kodaira M, Fujisawa T. Use of the GuideLiner catheter for aspiration thrombectomy in a patient with ST-elevation myocardial infarction with a large intracoronary thrombus. Cardiovasc Interv Ther, 2016; 31: 164-169.

5. Farooq V, Serruys PW, Mustafa AH et al. Forward and back aspiration during ST-elevation myocardial infarction: A feasibility study. EuroIntervention, 2016; 11: e1639-e1648.

6. Mitomo S, Naganuma T, Nakamura S, Tahara S, Ishiguro H, Nakamura S. Potential advantages of the GuideLiner catheter: insights from optical coherence tomography. Cardiovasc Interv Ther, 2015; Nov 3. [Epub ahead of print].

7. Capodanno D, Pratib F, Pawlowsky T et al. ClearWayRX system to reduce intracoronary thrombus in patients with acute coronary syndromes according to optical coherence tomography after abciximab intracoronary local infusion trial (COCTAIL): study rationale and design. J Cardiovasc Med, 2010; 11: 130-136.

8. Maehara A, Ben-Yehuda O, Ali Z et al. Comparison of stent expansion guided by optical coherence tomography versus intravascular ultrasound: The ILUMIEN II study. JACC Cardiovasc Interv, 2015; 8: 1704-1714.

9. Waterbury TM, Sorajja P, Bell MR et al. Experience and complications associated with use of guide extension catheters in percutaneous coronary intervention. Catheter Cardiovasc Interv, 2015; Dec 23. [Epub ahead of print].

10. de Man FHAF, Tandjung K, Hartmann M et al. Usefulness and safety of the GuideLiner catheter to enhance intubation and support of guide catheters: Insights from the Twente GuideLiner registry. EuroIntervention, 2012; 8: 336-344. 